\title{
Invasive Carcinoma Arising in Microglandular Adenosis of the Breast: A Case Report and Literature Review
}

\author{
Seung Won Oh, ${ }^{1}$ Hyo Soon Lim, ${ }^{1,}$ Jang Mi Baek, ${ }^{1}$ and Ji Shin Lee ${ }^{2}$ \\ ${ }^{1}$ Department of Radiology, Chonnam National University Medical School, Chonnam National University Hwasun Hospital, Hwasun-eup, Hwasun-gun, Jeollanam-do, Korea \\ ${ }^{2}$ Department of Pathology, Chonnam National University Medical School, Chonnam National University Hwasun Hospital, Hwasun-eup, Hwasun-gun, Jeollanam-do, Korea \\ "Corresponding author: Hyo Soon Lim, Department of Radiology, Chonnam National University Medical School, Chonnam National University Hwasun Hospital, \#322 \\ Seoyang-ro, Hwasun-eup, Hwasun-gun, Jeollanam-do, 58128, Korea. Tel: +82-613797112, Fax: +82-613797133, E-mail: nicolas-hs@hanmail.net \\ Received 2016 October 12; Revised 2017 February 16; Accepted 2017 July 06.
}

\begin{abstract}
Microglandular adenosis is an uncommon, benign breast disease considered to be a variant of adenosis. Breast carcinoma arising in microglandular adenosis has been reported. Because of its extreme rarity, its clinical and radiological features are not well known. We present a case of invasive carcinoma arising in microglandular adenosis of the breast in a 63-year-old woman with radiologic and pathologic findings. To our knowledge, this is the first case report with breast magnetic resonance imaging findings that shows the wide spectrum of the disease. Microglandular adenosis is important because it mimics carcinoma clinically and pathologically and breast carcinoma can arise in microglandular adenosis. When a core needle biopsy shows microglandular adenosis, complete excision should be considered to rule out the possibility of an associated carcinoma.
\end{abstract}

Keywords: Breast Neoplasm, Fibrocystic Breast Disease, Magnetic Resonance Imaging

\section{Introduction}

Microglandular adenosis (MGA) is an uncommon glandular proliferation of the breast, which is most often a microscopic lesion but may present as a palpable mass. MGA is important because it consists of proliferation of small round glands in fibrous or fatty mammary stroma that mimics carcinoma clinically and pathologically (1). Breast carcinomas arising in MGA have been reported, and MGA is thought to be a precursor of breast carcinoma (2). We report a case of invasive carcinoma arising in MGA of the breast with multimodality imaging findings and review of the literature.

\section{Case Presentation}

A 63-year-old woman came to our hospital because of a palpable mass in the left breast. On physical examination, an approximately $2 \mathrm{~cm}$ mass was palpated in the upper inner quadrant of the left breast. Skin changes and nipple retraction were not found. No axillary lymphadenopathy was palpable. She had no personal or family history of breast cancer.

Bilateral mammogram showed a 2-cm irregular mass with spiculated margins in the 11 o'clock position of the left breast (Figure 1). No calcifications were seen. On sonographic examination, an approximately 2-cm, irregular, indistinct hypoechoic mass was detected (Figure 2). An ultrasound-guided core needle biopsy was carried out on the mass, which revealed invasive ductal carcinoma in the left breast. Breast MRI was performed for preoperative staging. MRI showed a 2.6-cm irregular mass in the upper inner quadrant of the left breast, which showed heterogeneous enhancement with a delayed washout kinetic pattern after administration of gadolinium. Additionally, segmental, heterogeneous non-mass enhancement surrounding the biopsy proven malignancy was seen in the upper inner quadrant of the left breast, which was considered a suspicious finding (Figure 3). Fluorine $18\left({ }^{18} \mathrm{~F}\right)$ fluorodeoxyglucose (FDG) positron emission tomography/computed tomography (PET/CT) demonstrated hypermetabolism (maximum standardized uptake value, 8.7) of the breast mass (Figure 4) without other abnormal hypermetabolism. Segmental mastectomy was planned. During surgery, the surgical margins were positive for ductal carcinoma on frozen section and modified radical mastectomy was performed. Sentinel lymph node biopsy was performed, which showed no evidence of metastasis.

Grossly, a whitish-yellow colored, hard mass with illdefined borders measuring $3.0 \times 2.5 \times 2.0 \mathrm{~cm}$ was noted in the upper inner quadrant. Microscopically, small glandular structures infiltrating fibrous stromal septa or fatty stroma were observed in the peripheral area of the mass (Figure 5A). The glands were lined with a single layer of epithelial cells with round nuclei and abundant vacuolated cytoplasm (Figure 5B). Eosinophilic secretory mate- 


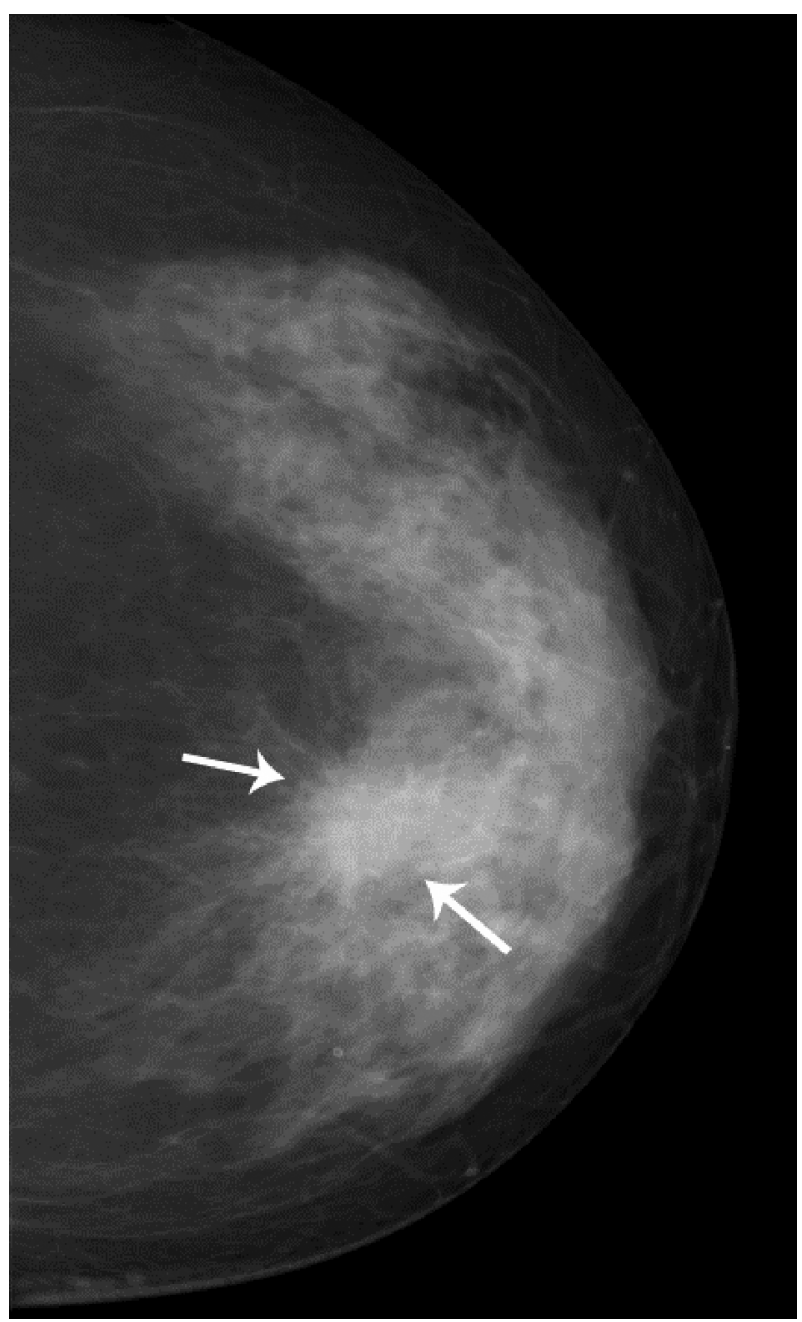

Figure 1. Left craniocaudal mammogram shows an irregular, equal-density mass with spiculated margins (arrows) in the upper inner quadrant of the left breast

rials were seen in the lumen of the glands. Immunohistochemically, glandular structures were surrounded by laminin-positive basement membrane. No myoepithelial cells were demonstrable with immunohistochemical staining for smooth muscle myosin heavy chain and p63 (Figure 5B, inlet). The epithelial cells were positive for S100 protein but negative for smooth muscle actin, estrogen receptor, and progesterone receptor. These findings were consistent with MGA. In some regions, ductal carcinoma in situ was admixed with glands of MGA(Figure 5C). The main mass was an invasive carcinoma of no special type, modified Bloom Richardson grade 3 (Figure 5D). On immunohistochemistry, the carcinoma cells were negative for estrogen and progesterone receptors and HER2-neu. This case was finally diagnosed as invasive carcinoma arising in

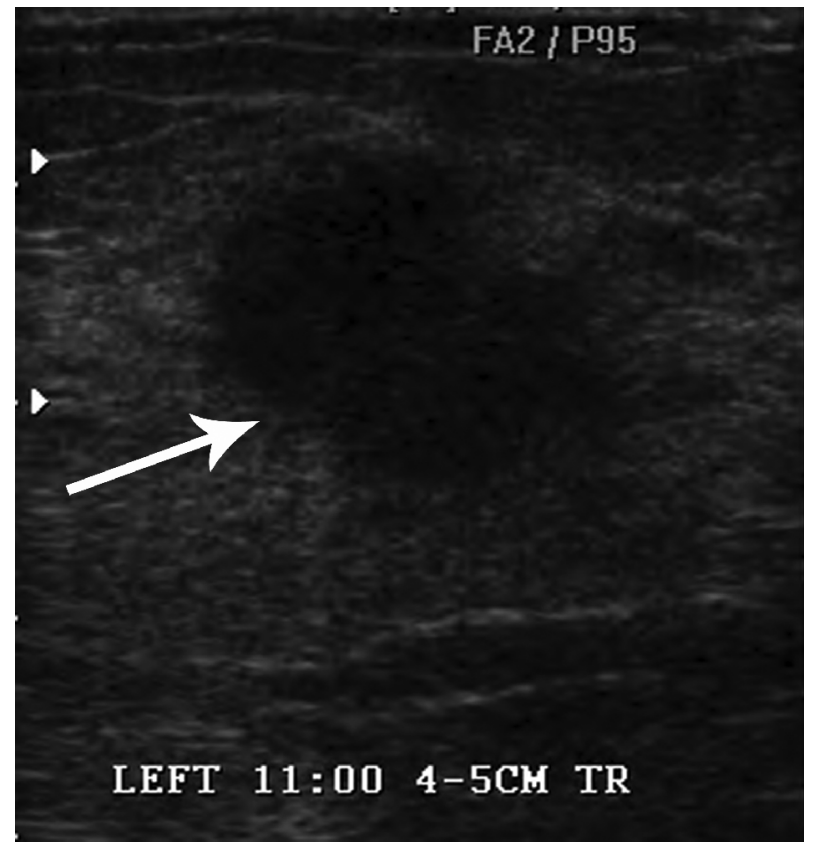

Figure 2. Ultrasound (US) image shows an irregular hypoechoic mass (arrow) at the 11 O'clock position of the left breast

MGA.

After surgery, six cycles of CMF (Cyclophosphamide, Methotrexate, and Fluorouracil)-based adjuvant chemotherapy were performed. After 32 months of follow-up, no recurrence was observed.

\section{Discussion}

MGA is an uncommon, benign breast disease considered to be a variant of adenosis. Diagnosis is frequently made by a pathologist, as the condition is often clinically asymptomatic. MGA is a rare epithelial lesion characterized by a proliferation of small round glands lined by a single layer of cuboidal epithelial cells with clear/vacuolated or eosinophilic cytoplasm and uniform nuclei (1). Unlike other intraductal proliferations and other forms of adenosis, the cells that line the glands do not have cytoplasmic protrusions or apical snouts, and myoepithelial cells are entirely absent, so the lesion may mimic welldifferentiated breast carcinomas, including tubular carcinoma (2).

Although MGA is benign in its uncomplicated form, a spectrum of lesions, ranging from MGA to atypical MGA and breast carcinomas arising in MGA have been reported $(3,4)$. The presence of atypical MGA in areas of transition between MGA and carcinoma suggests that MGA increases 

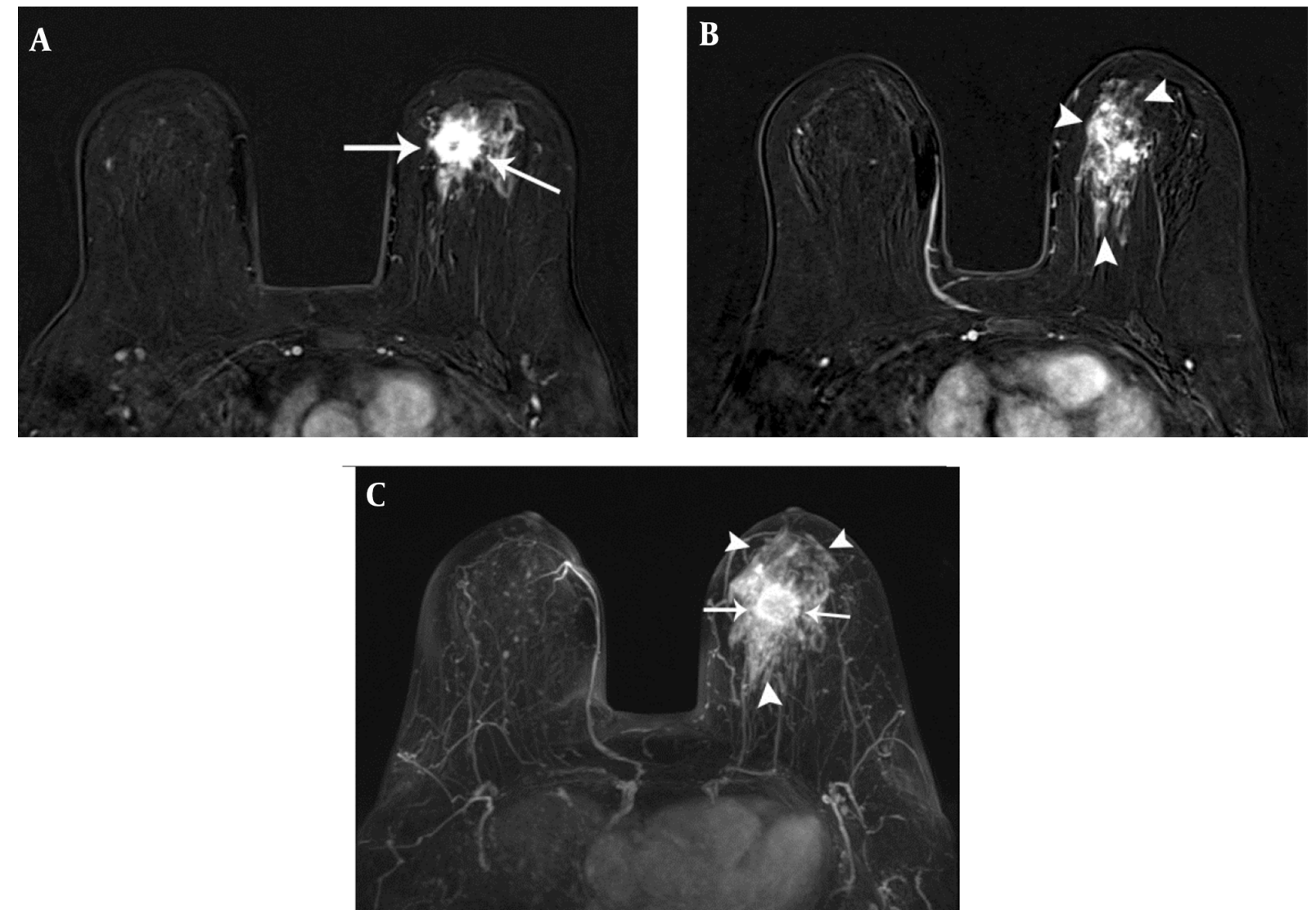

Figure 3. Early phase images of dynamic MRI (A and B) and a maximum intensity projection MR image (C) show a 2.6-cm irregular, heterogeneously enhancing mass (arrows) and segmental, heterogeneous non-mass enhancement (arrowheads) surrounding the mass in the upper inner quadrant of the left breast

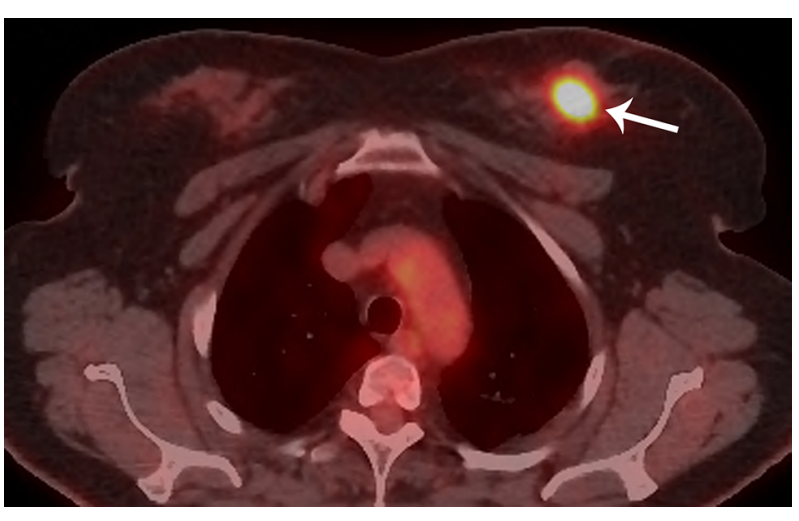

Figure 4. Axial fluorine $18\left({ }^{18} \mathrm{~F}\right)$ fluorodeoxyglucose positron emission tomography/computed tomography (FDG) (PET/CT) image shows markedly increased FDG uptake (maximum SUV, 8.7) indicative of hypermetabolism of the lesion (arrow)

the risk of developing carcinoma by serving as a precursor lesion (2).
Carcinoma arising in MGA has been reported in up to $27 \%$ of cases of MGA $(1,3,5)$. Carcinoma arising in MGA may show both in situ and invasive components. The basement membrane, which is usually preserved around the glands of MGA and atypical MGA, tends to be disrupted in invasive carcinoma arising in MGA (6). No special type (NST) is the most common type of carcinoma arising in MGA. Rare cases of adenoid cystic carcinoma, carcinoma with secretory differentiation, squamous metaplasia, chondromyxoid metaplasia, basaloid features, or a mixture of NST and matrix-producing carcinoma have been described in association with MGA $(3,4)$. The immunohistochemical profiles of carcinomas arising in MGA are mostly of a triplenegative phenotype (i.e. lack of ER, PR and HER2) and express $\mathrm{S} 100$ and resemble to that of MGA (3).

Because of its rarity, the radiological findings of MGA are not well known and there have only been a few reports on imaging findings of MGA and carcinoma arising in MGA. Although mammogram may reveal localized increased density, MGA was not detected on mammogram in 

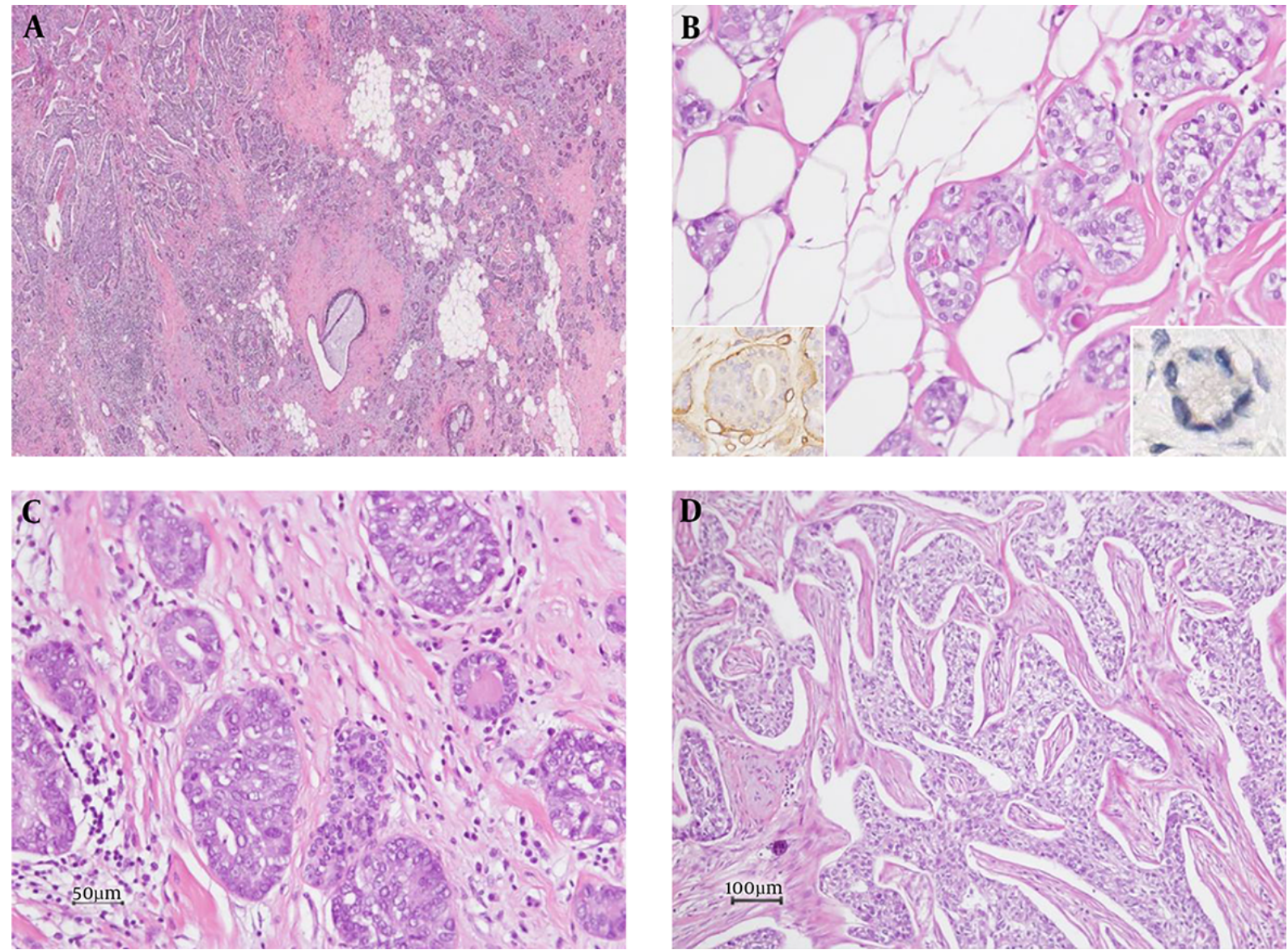

Figure 5. Photomicrograph of the histopathological specimen. A, Microscopic examination shows a tumor with an ill-defined border (H\&E staining, $\times 20)$. B, Microglandular adenosis composed of round glands lined by a single epithelial layer with laminin-positive basement membrane (left inlet, laminin immunohistochemistry) and lacking a myoepithelial layer (right inlet, p63 immunohistochemistry) are seen ( $\mathrm{H}$ and E staining, $\times 200$ ). C, Ductal carcinoma in situ admixed with microglandular adenosis is present (H and $\mathrm{E}$ staining, $\times 200)$. D, The main mass is an invasive carcinoma of no special type $(\mathrm{H}$ and $\mathrm{E}$ staining, $\times 100)$.

some studies $(4,7,8)$. Sonography revealed an ill-defined low echoic lesion or hypoechoic mass with irregular borders, discrete microlobulations, and angular margins (7, 8). There has been only one previously published case report on MR imaging findings of MGA, and breast MRI showed a small non-circumscribed mass with moderate early and delayed enhancement (8).

Carcinomas arising in MGA can be detected as masses on mammography and sonography $(4,9,10)$. Lee et al. reported that the lesion was an irregular shaped, hyperechoic nodule with indistinct margins and pleomorphic internal microcalcifications (9). In one study, the lesions were seen as irregular or lobular hypoechoic masses (10). In our case, mammogram and ultrasonography showed an irregular mass without calcifications. On breast MRI, not only an irregular mass but also segmental, non-mass enhancement surrounding the mass was evident. Patholog- ically, the irregular mass was confirmed as invasive carcinoma of no special type and most of the segmental, nonmass enhancement surrounding the mass was confirmed as MGA to atypical MGA. Although ductal carcinoma in situ was admixed with glands of MGA in some regions on histopathology, we could not differentiate ductal carcinoma in situ from MGA or atypical MGA on breast MRI. To our knowledge, this is the first case report of breast MRI findings that shows the wide spectrum of carcinoma arising in MGA.

When a core needle biopsy shows MGA, complete excision should be done and the excision specimens must be sampled thoroughly to achieve margins negative for the lesion and rule out the possibility of associated carcinoma. The treatment of carcinoma arising in MGA depends on the stage of the disease. It is important to assess the extent of the disease before surgery because MGA and atypical MGA 
may recur and carcinoma arising in MGA may occur if the affected area is incompletely excised $(1,4)$. Resetkova et al. reported a case of carcinoma arising in MGA that recurred 10 years after breast conservation surgery with incomplete resection of MGA, suggesting the importance of attaining complete excision to reduce the likelihood of recurrent carcinoma (5).

Breast MRI may be useful in assessing not only the extent of carcinoma but also the extent of MGA and atypical MGA, which is frequently occult on mammography. In our case, MGA and atypical MGA were seen as segmental non-mass enhancement surrounding the carcinoma. Various benign, high-risk, and malignant diseases in the breast can show non-mass enhancement on MRI. The differential diagnosis for non-mass enhancement on MRI includes pseudoangiomatous stromal hyperplasia, apocrine metaplasia, flat epithelial atypia, intraductal papilloma, radiation effect, atypical ductal hyperplasia, radial scar or complex sclerosing lesion, ductal carcinoma in situ, invasive ductal carcinoma, and invasive lobular carcinoma. Mastopathic changes such as adenosis, hormonal stimulation, inflammatory changes, and focal or diffuse fibrocystic changes are the most common benign causes of nonmass enhancement. Combined analysis of distribution, kinetics, and internal enhancement patterns of non-mass enhancement will facilitate better characterization of lesions $(11,12)$. More studies are needed to set up the characteristic MR imaging findings of MGA, atypical MGA and carcinomas arising in MGA, which can help in assessing the extent of the disease.

In summary, we report a case of invasive carcinoma arising in MGA of the breast. Although MGA is generally benign, complete excision should be considered to rule out the possibility of an associated carcinoma when a core needle biopsy shows MGA.

\section{Footnotes}

Authors' Contributions: Seung Won Oh drafted the manuscript. Hyo Soon Lim drafted and performed critical revision of the manuscript for important intellectual content. Jang Mi Baek was responsible for acquisition of data. Ji Shin Lee made the pathological diagnosis and helped the draft. All authors contributed to the content and approved the final version of the manuscript.
Declaration of Interest: All authors declare that they have no conflict of interest.

Financial Disclosure: None declared.

Funding/Support: None declared.

\section{References}

1. Rosen PP. Microglandular adenosis. A benign lesion simulating invasive mammary carcinoma. Am J Surg Pathol. 1983;7(2):137-44. [PubMed: 6859388].

2. Eusebi V, Foschini MP, Betts CM, Gherardi G, Millis RR, Bussolati G, et al. Microglandular adenosis, apocrine adenosis, and tubular carcinoma of the breast. An immunohistochemical comparison. Am J Surg Pathol. 1993;17(2):99-109. [PubMed: 8422116].

3. Koenig C, Dadmanesh F, Bratthauer GL, Tavassoli FA. Carcinoma Arising in Microglandular Adenosis: An Immunohistochemical Analysis of 20 Intraepithelial and Invasive Neoplasms. Int J Surg Pathol. 2000;8(4):303-15. doi: 10.1177/106689690000800409. [PubMed: 11494007 ].

4. Khalifeh IM, Albarracin C, Diaz LK, Symmans FW, Edgerton ME, Hwang RF, et al. Clinical, histopathologic, and immunohistochemical features of microglandular adenosis and transition into in situ and invasive carcinoma. Am J Surg Pathol. 2008;32(4):544-52. doi: 10.1097/PAS.ob013e31815a87e2. [PubMed: 18300793].

5. Resetkova E, Flanders DJ, Rosen PP. Ten-year follow-up of mammary carcinoma arising in microglandular adenosis treated with breast conservation. Arch Pathol Lab Med. 2003;127(1):77-80. doi: 10.1043/0003-9985(2003)127<77:TYFUOM>2.0.CO;2. [PubMed: 12521372].

6. Salarieh A, Sneige N. Breast carcinoma arising in microglandular adenosis: a review of the literature. Arch Pathol Lab Med. 2007;131(9):1397-9. doi: 10.1043/15432165(2007)131[1397:BCAIMA]2.0.CO;2. [PubMed: 17824796].

7. Kim DJ, Sun WY, Ryu DH, Park JW, Yun HY, Choi JW, et al. Microglandular adenosis. J Breast Cancer. 2011;14(1):72-5. doi: 10.4048/jbc.2011.14.1.72. [PubMed: 21847399].

8. Sabate JM, Gomez A, Torrubia S, Matias-Guiu X, Alonso C, Pericay $\mathrm{C}$, et al. Microglandular adenosis of the breast in a BRCA1 mutation carrier: radiological features. Eur Radiol. 2002;12(6):1479-82. doi: 10.1007/s00330-001-1174-4. [PubMed: 12042957].

9. Lee YH, Dai YC, Lin IL, Tu CW. Young-aged woman with invasive ductal carcinoma arising in atypical microglandular adenosis: a case report. Pathol Int. 2010;60(10):685-9. doi: 10.1111/j.1440-1827.2010.02577.x. [PubMed: 20846267].

10. Choi JE, Bae YK. Invasive breast carcinoma arising in microglandular adenosis: two case reports. J Breast Cancer. 2013;16(4):432-7. doi: 10.4048/jbc.2013.16.4.432. [PubMed: 24454466].

11. Chadashvili T, Ghosh E, Fein-Zachary V, Mehta TS, Venkataraman $S$, Dialani V, et al. Nonmass enhancement on breast MRI: review of patterns with radiologic-pathologic correlation and discussion of management. AJR Am J Roentgenol. 2015;204(1):219-27. doi: 10.2214/AJR.14.12656. [PubMed: 25539260].

12. Giess CS, Raza S, Birdwell RL. Patterns of nonmasslike enhancement at screening breast MR imaging of high-risk premenopausal women. Radiographics. 2013;33(5):1343-60. doi: 10.1148/rg.335125185. [PubMed: 24025928]. 